Quant aux impurelés de la bauxite, elles sont réduites au moment de l'azoturation (l'oxyde de fer, par exemple, donne du fer métallique) el forment, après atlaque par la lessive alcaline, un résidu lourd, facilement séparable de la liqueur par une simple décantation.

D'après le $\mathrm{D}^{\mathrm{r}}$ Serpek, Ja transformation de la bauxite en alumine coûte r $25 \mathrm{fr}$. 25 par tonne d'alumine pure, celle du nitrure en alumine ne dépasse pas $63 \mathrm{fr}$. 80 .

La production mondiale de l'aluminium, qui est passcé de 35 ooo tonnes en rgr à 65 ooo tonnes en IgI2, est appeléc à se développer rapidement. On peut affirmer aujourd'hui que ce "petit métal " deviendra, dans l'avenir, un nétal d'une consommation comparable à celle des autres mélaux communs, comme le zinc par exemple, de l'ordre de 200000 tonnes, en tenant compte de la différence des densités.

M. Matroxon base son affirmation sur les deux rajsons suivantes. Toul d'aboird, la résistance chimique du métal augmente rapidement quand ses impuretés diminuent ; là. comme dans bon nombre de réactions chimiques, ce sont les impurclés qui facilitent leur mise en marche ; avec l'aluminium, cette particularité est tout à fait accuséc. Et toul nouveau progrès réalisé dans la diminution des impurclés aura pour but d'améliorer les qualités chimiques du mćlal. En second lieu, les fabricants d'aluminium étaient jusqu'ici sans lien commun avec les lamineurs et tréfileurs du métal ; il en résultait un manque d'entente et d'initiative pour développer et multiplier les applications de l'aluminium. Aujourd'hui; gràce à l'accord qui s'est établi entre les productcurs franģais d'aluminium el dont le procédé Senper fut la cause, la dualité précédente est appelée à disparaître eu mèmo temps que la concentration des efforts al leur unité de direction faciliteront singrulierement le développement de ce métal. On peut done aflirmer que les espé- nces que Sainte-Claire Deville avaient fondées sur l'alurinium sont à la veille de devenir des réalités, et que co nouveau mólal peul ètre considéré comme " ayant alteinl sa majorité "s.

D'après la production de rgra, 65 ooo tomnes, on peul calculer une obtention simullanée de 132 ooo tomes de sulfile d'ammoniaque ; et le jour où celle production antuil fuadruplé, on alteindrait 528 ooo tonnes de sulfate.

Mais le procédé Serpek peut fonclionner, non sculement comme producteur dalumine avec l'ammoniaque commo produit secondaire, mais également comme producteur d'ammoniaque, la même ahmine rentrint loujours dians la fabrication.

La décomposition de l'arolure, fans ce dernich cas, est entcore simplifice, car il n'est plus néressate d'oblenir do l'alumine pure : au conl'alire, il fanl comserver it celle-ci ses inlpurclés calalysantes ol par consépluent lopéralion doviont plus économique. (d suivre).

\title{
REVUE DES SOCIÉTÉS SAVANTES ET DES PUBLICATIONS TEGHNIQUES
}

\section{ACADÉMIE DES SCIENCES}

\section{ÉTUDES GLACIOLOGIQUES}

La vitesse des glaciers en hiver et l'inanité de la théorie thermique de leur progression. Note de M. J. Val cot, présentée par le Prince Roland Bonaparte. Séance du 23 juin 1913.

La vilesse annuelle de progression des glaciers a fait l'objet de nombreuses études; mais le rapport entre la vitesse d'été et la vitesse d'hiver est totalement inconnu, les expériences qui ont été faites ayant été toutes viciées par des erreurs de technique.

La plupart du temps, on s'est contenté de suivre et de mesurer la marche d'un bloc erratique pendant une longue période ; mais M. Vallot a démontré que les changements de pente rencontrés par le bloc dans son excursion produisent des changements de vitesse correspondants qu'on a pris pour l'influence des saisons.

Le seul procédé légitime consiste à mesurer successivement pendant l'été, et ensuite pendant l'hiver, la vitesse de la même région du glacier, en ayant soin de rétablir les jalons rigoureusement sur la même ligne.

Ce procédé a été employé par Tyndall, il y a cinquante ans ; mais son expérience a ćté vicice par cette circonstance que les deux mesures ont été faites à plusieurs années d'intervalle, au cours de la décroissance rapide du glacier. Or $M$. Vallot a démontré que la vitesse varie avec les crues et que chaque diminution de $\mathrm{r} \mathrm{m}$. dans le niveau de la Mer de Glace amène une diminution de $2 \mathrm{~cm}$. par jour dans la vitesse, de sorte que les expériences de Tyndall ne sont pas comparables entre elles.

M. Vallot a entrepris de nouvelles mesures, pour lesquelles il s'est efforcé d'éviter les causes d'erreurs qui affectaient les expériences de ses devanciers.

En été r912, il a établi en travers de la Mer de Glace, au Montanvert, une ligne droite formée d'une vingtaine de pierres numérotées, distantes cntre elles de $20 \mathrm{~m}$. environ. Ces repères mobiles ont été alignés an théodolite, et leurs distances ont été chaînées. La ligne avait une longueur de $350 \mathrm{~m}$. et occunait $1 \mathrm{a}$ parlic médiane du glacier, partie dont les divers points onl des vilesses peu différentes. Lexpérience a duré du 6 aon̂t au 7 scoptembre, soit 32 jours, au bout desquels de nouvelles mesures onl. permis de connaitre l'espace parcouru par chacun des repòres mobiles ainsi disposés.

L'expérience a été repriso au conrs de la saison froide qui at suivi immédiatement. Une ligne de piquets en hambous plantix dans la neige a élć élablic rigonrensement an môme perint he 29 décembre rgr\%. La position des repères mohiles a ćlé mesurie le même jour et les mesures ont été reprises le / février, 37 jours après. - Le compte rendu contient un tableau donnant la position des repères mobiles au commencernent de chaque expériche: et la vilesse de chacun d'eux en centimètres par jour.

La moyenne donue $2 /, 6$ pour l'eté at $2,4,3$ pour l'hiver, en centimèlres de vilesse par jour. L'erreur à craindre ćtant do $1 \mathrm{~cm}$. , la concordance est remarquable. Le détail des chiffres monlers qu'il ne s'agil pas d'un hasard de moyennes, mais bien de chiffres semblables sur tous les points de la ligne. On peut dire que la vitesse du glacior n'est pas sensiblement différente en blé al. en hiver.

Depuis trois siècles (Scheuchzer, r 708 ) le sujet est à l'élude el les glaciologues sont divisés sur la question de savoir si le glacier descend sur la pente uniquement par son poids on s'il progresse par dégel, avec introduction d'eau suivic de regel dans ses fissures. Les expériences de M. Vallot à la Mer de Glace ont ćlabli divers caractères qui assimilent le glacier à un fleuve coulant dans son lit : le glacier coule parallilement à ses rives et non en éven. tail ; la vilesse de chague point varie en raison de la pente; la vilesse augmente avec les crues, etc. D'un autre côté, Forel et Hagenbach ont établi que l'existence des fissures capillaires ctait limitée à l'écorec du glacier et que Ia masse était imperméable. M. Vallot a signalé le même fait dans le tunnel du sommet du Mont Blanc.

Il a démontré que la partie du glacier muverte de moraines progresse avec la même rapidité que la partie découverte, bien que beaucoup moins échanffée. Il a démontro aussi que Jes glacier. 
supérieurs, qui pourtant ont un mouvement très caractérisé, ne peuvent pas avoir des alternatives de dégel et regel, puisque la rariation diurne ne pénètre pas à plus de $1 \mathrm{~m}$. de profondeur et que plus bas le glacier demeure au-dessous de zéro. Il a fait voir ensuite que la variation annuelle ne pénètre pas au dela de 6 à 7 mitres de profondeur, et que la masse des glaciers de grande illitude se trouve à des températures uniformes trop basses (jus(qu'à $-13^{\circ}$ et $-15^{\circ}$ ) pour laisser supposer l'introduction de l'eau même s'il existait des fissures capillaires.

Un seul indice de l'action de la chaleur restait encore, c'était l'augmentation de la vitesse en été. Daprìs les chiffres donnćs ci-dessus, il paraît devenu certain que celte augmentation de vitesse n'existe pas. Toutes les objections sont tombées une à une. La théorie thermique s'écroule et paraît définitivement ruinće. Aucme variation calorifique, soit diurne, soit annuelle, ne peut pénétrer la masse interne du glacier, et celui-ci peut être considéré comme un fleuve qui s'écoule dans son lit sous la seule influence de la pesanteur.

\section{PHYSIQUE DU GLOBE}

Formule barométrique simplifiée pour la mesure des altitudes. Note de M. Alphonse BengET, présentée par M. Ch. Lallemand, séance du 30 juin 1913 .

La mesure des hautcurs à l'aide du baromètre est, le plus souvent, le seul moyen qui soit à la portée des géographes voyageurs. Pour faciliter cette mesure, les constructeurs ont réalisé des baromitres anćroïdes dont le cadran porte des divisions égales devant donner firectement les altitudes atteintes; ces instruments (a l'exception de ceux du colonel Goulier, quand on $y$ lit directement les altitudes, penvent conduire à des erreurs notables, dès que les différences de niveau sont un peu fortes.

La meilleure manière d'utiliser le baromètre à la mesure des altiludes consiste à observer la hauteur de mercure aux deux stalions dont on veut connaître la différence de niveau, ou, si l'on dispose d'un baromètre anéroide, les chiffres du cadran qui indiquent celle hauteur. Il faut connaître également les nombres qui indiquent les températures de l'air aux deux stations, les tensions de la vapeur d'eau et, si l'on se sert d'un baromètre à mercure, les températures qu'indique le thermomètre fixé à l'instrument (avec les baromìtres anéroïdes compensés ces derniers nombres ne sont pas nécessaires).

Les lectures des hauteurs $\mathrm{I}_{0}$ et $\mathrm{H}$ de la colonne de mercure aux deux stations, ainsi que les autres observations, doivent être failes, autant que possible, simultanément. A l'aide des nombres observés, on calcule la différence de niveau $Z$ de deux stations a l'aide de la formule logarithmique bien connue que donnent les Tables méléorologiques internalionales.

Le calcul de $Z$ à l'aide de celte formule est long et pénible. Pour le faciliter, on a construit des Tables dans lesquelles une fitrlis des lemes qui y figurent sont tout calcules pour les diverses valeurs des variables d'observation; mais il faut posséder ces lables el, même avec lour aide, les calculs exigent une cerlaine habilude.

Si l'on se contente d'une approximation de $\mathrm{x} / 500$, bien suffisanle quand on emploie le baromìte anéroïde, qui 11 'en comporte pas de plus grande, on peul se servir d'une formule plus simple, dont M. Nphonse Berget a délerminé le coefficient numérique, pour loutes les différences de niveau comprises entre aéro et $2500 \mathrm{~m}$. environ. Celle formule ne demande ni l'emploi des Tables de lograrithmes, ni celui des Tables spéciales, et n'exige que des opérations simples d'arithmétique.

Lal formule proposée est : $Z=30 \frac{D}{h}(t+269)$,

dans laquelle $Z$ est la différence de niveau cherchéc, D la différence des pressions Jues sur le baromètre aux deux stations, $h$ la valeur moyenne de la pression à ces deux endroits et $t$ la temperature moyenne.

Les calculs sont, on le voit, fort simples. Malgré cela, la pré. cision donnéc par la formule est amplement suffisante, comme le montrent les exemples suivants, où les résultats qu'clle fournit sont comparis à ceux que donne le long calcul de la formule logarithmique. a. Altitude du Guanaxuato, déterminée par de Humboldt. Les données sont :

$\mathrm{II}_{0}=763^{1 \mathrm{~mm}} \mathrm{I} 5, \quad \mathrm{H}_{2}=600^{\mathrm{mm} 9} 9^{5}, \quad \theta_{0}=25^{\circ} \mathrm{r} \quad \theta_{1}=21^{\circ} 3$.

La formule ( $\mathrm{r}$ ) donne $\mathrm{Z}=2083^{\mathrm{m}} 63$ (la formule logarithmicque donne $\mathrm{Z}=2084^{\mathrm{m}}$ ).

b. Altilude du Puy-de-Dôme. — Les données sont :

$\mathbf{H}_{0}=7^{38^{\mathrm{mm}} 7}, \quad \mathrm{H}_{1}=647^{\mathrm{mm}} \mathrm{s}, \quad 0_{0}=1 \mathrm{I}^{\circ} 5, \quad 0_{1}=7^{\circ} \mathrm{I}$

La formule (r) donne : $Z=\log ^{\mathrm{w}}$ (la formule logarithmique donne : $\mathrm{Z}=\mathbf{I} \circ 89^{\mathrm{m}}$ ).

c. Exemple tiré des Tables météorologiques internutionales, p. A.42. - Les domnées sont :
$\mathrm{H}_{0}=7^{3} 9^{\mathrm{mm}} 7^{3}$,
$\mathrm{H}_{1}=583^{\mathrm{mm}} / 4 \mathrm{I}$
$l$ (moyen $)=+8^{\circ}$.

La formule $(r)$ donne : $Z=\mathbf{I} 963^{\mathrm{m}}$ (la formule logarithmiqu donnc $: Z=I 965^{\mathrm{m}}$ ).

d. Exemple tiré des Instructions météorologiques. - Les don. nées sont :

$\mathrm{H}_{0}=7 \mathrm{I}^{\mathrm{mm} / 4}, \quad \mathrm{H}_{1}=543^{\mathrm{mm}} 2, \quad 0_{0}=17^{\circ} 5, \quad \theta_{1}=2^{0} \mathrm{I}$.

La formule ( $\mathrm{I}$ ) donne : $\mathrm{Z}=2339^{\mathrm{m}} 6$ (la formule logarithmique donne : $\left.\mathrm{Z}=2338^{\mathrm{m}}\right)$.

On voit donc que la précision de la formule ( 1 ) justifie son chiploi ; elle a pour clle sa très grande simplicité el la laciilí des calculs qu'elle comporte et qui la rendront précicuse pour tons ceux qui ont à faire des nivellements rapides en monlagne. Elle ne doit pas être employéc au calcul de différences de niveall supéricures à 3 ooo mètres.

\section{ÉLECTRO-MÉCANIQUE}

Sur les champs magnétiques obtenus avec un électroaimant muni de pièces polaires en ferrocobalt. Note de M. Pierre Weiss, transmise par M. J. Violle, séance du 30 juin 1943 .

L'étude des ferrocolalts, faile sous la direction de l'auteur par II. A. Preuss (Thèse, Zurich, rgra), a montré que le fer qui possòde $x$ magnétons par alome, forme avoc le cobalt, qui en a 9 , un composé défini $\mathrm{Fe}^{2} \mathrm{Co}$ de r 2 magnétons par atome. Si l'on tient compte de la différence des poids atomiques, de celle des tempí. ratures de perte du ferromagnélisme et do celle des densités, on trouve que le ferrocobalt a une aimantation à saturation de ro pour roo supérieure à celle du fer, à la température ordinaire.

En attendant que l'industrie loumisse le ferrocobalt, M. de Frendenreich a réalisć par les moyens du laboratoire celte préparation, assez délicate lorsqu'il s'agit d'obtenir des pièces de dimensions notables sans trop de soufflures. Avec les matières premières : for de Suède et cobalt industriel à 98,5 pour roo, les expérimentateurs ont obtenu dans différentes fontes un gain de 9 pour roo el te 9,7 pour roo, au lieu des so pour roo de l'allinge de composition exacte. Le lingot qui donnait 9 pour roo était le plus compact dt a servi à former les pièces poliaires. Faute de matière suffisante pour les faire tout entiore en ferrocobalt, on s'est borné à lis munir de poinles de ferrocobalt.

Les champs obtenus avec ces pièces polaires et avec d'aulres, exactement de mêne forme, en f'er de Suìde, ont été mesurécs all moyen de la même méthode.

Les mesures ont indiqué que dans lous les cas le gain par lie forrocobalt a été voisin de 5 pour roo. Même le remplacenent partiel du fer par le ferrocobalt est aussi effeace que l'angmet tation de la puissance dépensée dans le rapport de 1 à 4 .

Une partie de ces mesures se rapportent à un entrefer de i 4 millimètres cubes dans lequel on peut placer une étincelle, une pe tite flamme ou un milieu absorbant. Dans un espace de o $\mathrm{mm}^{3} \mathrm{~b}$, suffisant pour loger un grain de radium, on eût obtenu 75000 gg.

L'électro-aimant est bobiné avec I ooo tours de lube de cuive parcouru par un courant d'eau, le métal servant de conducteur b́lectrique. Pour pouvoir faire passer la quantilé d'ean nécessairi à la réfrigération, le tube est divisé en ro sections, en dérivalion pour le courant d'eau et en séric pour le courant élcctrique. lo débit d'caut est 6 litres par minulc et l'échauffement maximum $50^{\circ}$. L'eau froide étant amenée dans la première couche de chaque bobine, le noyau reste froid. L'appareil altcint sa tumpérature de régime en 2 minutes el la garde indéfiniment. 


\section{REVUE DES PERIODIQUES ETRANGERS}

Balance thermique dans les chaudières, par $\mathrm{L}-\mathrm{S}$. llarks, Pouver, 14 janvier 1913, p. 42 à 47.

L'article fournit un procédé simple et clair pour le calcul de la "balance thermique " (c'esl-it-dire du décompte des calorics) d'un générateur de vapeur, en hitilisant les résultats dun essai ordinaire de production.

Les calories fournies par le combustible peuvent prendre une les cing directions suivantes :

$I^{\circ}$ Chaleur employée à l'élévation de la tempéralure de l'eau al a la production de la vapeur.

$2^{\circ}$ Chaleur non développée par suite d'une combustion incom. nite ; cette chaleur peut se diviser en trois calégories :

a) Chaleur de combustion du charbon (ou de toule autre malière combustible) tombé dans le cendrier et cnlcré avec les cscarbilles ;

b) Chaleur de combustion de l'oxyde de carhone qui s'en va par la cheminée d'appel ;

c) Chaleur de combustion des produits hydrocarlonés qui s'en rout non hrulés par la cheminéc d'appel.

$3^{\circ}$ Calories véhiculées sous la forme de chaleur sensible des gaz chands dégagés à la chemince (a l'exchsion de la vapeur d'cail).

$4^{\circ}$ Calories véhiculées sous la forme de vapeur d'eau dégagée it la cheminée en mème temps que les autres gaz provenant de. la combustion. Cette vapeur d'ean provient de trois sources disfinctes et il faut distinguer :

a) celle qui résulte de la combusion de l'hydrogène contenu dans la houille;

b) celle qui provient de I'humidité de la houille ;

() celle qui provient de l'humidite de l'air.

$5^{\circ}$ Chaleur perdue par radiation et conducljon, ést-atdire toule relle dont il n'a pas été tenu compte jusqu'ici.

Le probleme de la détermination de la balance thermique d'un générateur consiste dans la détermination des chiffres correspondant aux catégories $x, 2,3,4$. I a chaleur perdue par radiation et conduction se détermine par différence.

L'article fournit les méthodes permettant d'offectuer cette détermination, des barêmes étant employés pour simplifier les alculs dans le cas des paragraphes 3 ct 4 .

Dans un exemple d'application do cette méthode il est donné les chiffres suivants :

Chalcur fournic à l'eau d'alimentation ........... perdue par non combustion du charbon... $75,3 \%$ perdue par le dégragement de CO.......... entraînée par les gaz chands (autres que la vapeur d'eau) $\ldots \ldots \ldots \ldots \ldots \ldots \ldots \ldots$. entraince par la vapeur deau provenant de la combustion de l'hydrogène de la houille entrainéc par la vapeur d'eau provenont do

l'humidité de la houille.............. entrainée par la vapeur d'cau provenant de

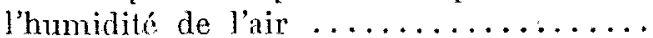
perdue par radiation et conduction.........

Instruments de mesure à très grande longueur d'échelle. Electrical Engineer, 9 janvier 1913, p. 21. Electrical Revieu", 10 janvier 1913 , p. 52 .

Deseription du nouvel apparcil établi par J.-W. Record, de la Record Electrical $\mathrm{C}^{\circ}$. Ces ampèremètres et voltmètres ont une chelle correspondant à un angle de déplacement de l'index de $300^{\circ}$, mais ne consomment pas plus de puissance que ceux qui ne posseddent qu'un angle de $90^{\circ}$. Les pièces polaires sont de forme annulaire. Un des pôles comporte deux disques, l'autre un seul placé entre les deux précédents. Un faible entrefer est ménagé entre les 2 systèmes.
Par ce disposilif, la bobine est libre daccomplir une rotation presque entière.

Les entrefers sont en parillele, ce qui réduit la reluchance du circuit magnétique.

L'aimant permanent sert de support prineipal pour les parties mobiles de l'appareil. La longueur inutilisée des conducleurs du cadre cst reduite au minimum of l'appareil n'a qu'me tris fable resistance.

Limortissoment est perduil par les courants induits dans le Lrati.

La vérification de la forme des courbes de f.e. $m$. C.-M. DAvis. American Instilute Procecdings, 32, p. 2.35-241. Fevrier 1913.

Les procédés usités achullement el recommandés pour la várification de la forme plus on moins simusödale des courbes de f. e. m. sont sujets a plusicurs objedions dout la principale est la necesite de lemploi diun oneillographe. Le systeme frophei est base sur lo changement de la réactane diun condensaleur gui accompagne la déformation de l'onde.

Les avantares de celte mithode sonl los suivanls :

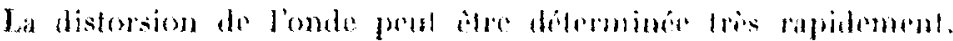
Ginfluence des harmoniques, en tanf qu'ordere al amplilude, as

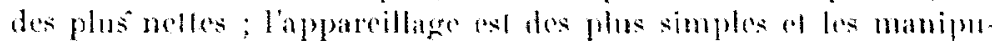
lations sont trìs faciles.

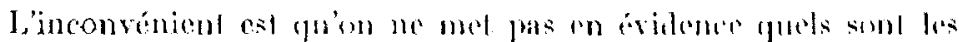
hamoniques prísents.

Le rapport de la reatetare offerte par le coudensalenr aver

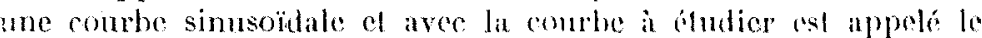
rapport on "coefficionl de distorsion".

On le mesure en appliefunt suecessivement la difference do potentiel à étudier sur un rirenit eontenant le condensateur ave une rúsistance induelive considérable en seirie, ef sur le conden. sateur senl.

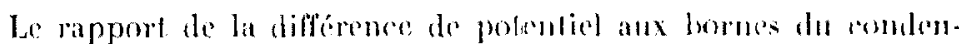
sateme à l'intensite, détermine dans les deux mas da rénclance.

I a mesure relative à la comble de f. c. m. d'un généraleur doil so faim à vide, car la courbe en charge n'est pas définic.

Plusieurs relerís ì l'secillographe, affoctués récemment sonl donnés, accompagnés du coefficient de distorsion calculé, d aussi de la décomposilion ru harmoniques.

En appendice. - Formules theoriques et tables pour calculor la distorsion admissible.

L'auleur propose d'introduire ee procédé dins la réglementa. tion de J'American Institule, et indique en conséquence comment derraient être libellées les parties correspondanles de cetle réglementation.

Les effets de la déformation de l'onde sur le matériel électrique, par P.-M. L Ixcoss. American Inslilule Proceedings, 32. p. 377-388. Fevrier 1913

Les règles actuellement en vigneur imanant de l'American Ins. libule of Electrieal binginoers, an sujet te la formo de l'onde tr f. c. In., ne sont pas satisfaisantes car elles ne prinalisent pris sulfisamment la prísence des harmonijues.

L'autenr examine les consépuences que prisente l'iearl par rapport a la forme simusojulale de l'ondr de f. o. m. of si repor. russion sur la forme de l'intensilé dans les différents circuits qui penfent se présenter : aves risistance, avec inductance, aver calpacite.

Il examine ensule ce gui se passe quand deux fores bleclor. motrices de forme differente se fromsent mises an parallible of re qui en rísulte pour l'intensité.

Il montre comment, en cerlains cas, il peut résulter une intensilé trìs déforméc, on des courants de circulation très intenses, de l'accouplement de machines synchrones possédant des ondres de f. c. m. tris dissemblables.

Il est donné plusieurs exemples de doformations excessives de la f. c. $m$. et du courant. Une étude est faite des crreurs qui pewyent s'ensuivre dans les phasemetres et autres appareils. 
La mesure des températures dans les machines électriques à mouvement de rotation. L. W. Cutвв, E. J. Cintre et O. W. A. Cerrixa. Comptes rendus de American Institute of Electrical Engineers, p. 583-597. Février 1913.

Le mémoire renferme une discussion des avantages et des inconvénients des méthodes ríglementaires (Standard) el des différents autres procédés employés pour la détermination d s températures des machines.

La température maxima affectant l'isolement est le factear qu'il convient de déterminer dans les mesures portant sur la tempéralure des machines.

Liomplus du thermonètre, on la méthode par l'élévation de lit résistance, rencontrent de fréquentes contre-indications et, souvent, conduisent, si on ne tient pas un compte très soigneux des conditions locales, à des résultats erronés.

L'emploi des méthodes basées sur le couple thermo-électrique est disculé en détail, car il est d'une application assez récente.

L'auteur donne rne description de l'emploi de tels couples ilemo-électriques et indique les résultats que ce procódé a permis d'ohlinir en ce qui concerne la distribution de la chaleur dans un gencialeur électrique.

Elude est faite ensuite de la question de la température de l'enreinle. La conclusion est la suggestion d'un certain nombre de modifications of d'additions ì la réglementation concernant les essais de réception les machines.

Détermination des pertes supplémentaires dues a la charge pour les machines a mouvement de rotation, par E. M. Olin et S. L. Heunersox. Compte-rendu de l'American Institule of Electrical Engineers. 32. p. 521-j40. Férrier 1913.

Io mémoire décrit les essaj qui ont élé effectuís pour déter. miner les "pertes supplementaires dues à la charge " pour les moleurs et génératenrs à commant continu, les générateurs à courant alternatif, les moleurs synchrones, les moleurs asynchrones.

On entend sous ce terme la différence entro l'ensemble des pertes róclles en charge, al la somme des pertes caleulées d'après les résullals des mesures cffectuées dans l'essai à vicic. Il est communiqué des déterminations trìs précises des pertes dans les conditions pratiquement équivalentes à celles qui existent en charge.

Les perles supplémentaires résullent de l'augmentation de la dissipation d'énergie dans l'armature par suite de la distorsion du champ et, en second lieu, des courants parasites engendrés dans les conducteurs do l'armalure par les champs provenant du courant de travail lui-même.

Les facteurs de correction proposés s'appliquent à la somme de la perte $\mathrm{RI}^{2}$ d'armature et de la perte à vide de cette armalure. Quelques essais conduits au laboratoire ont fait ressortir l'existence d'une valeur uniforme pour le facteur de correction.

Les auleurs recommandent done les valeurs suivanles pour le facteur de correction proposé :

Fraction de la charge ............ $1 / 2$ $3 / 4$ I $5 / 4$

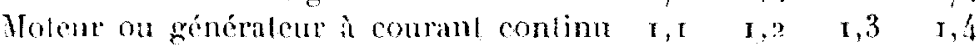
Générateur ì courant alternatif el Moteur synchrone (l'un ef l'autre avec con-

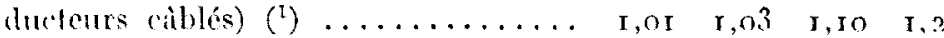

Les installations de la Compagnie de distribution d'énergie de Victoria Falls et du Transvaal, par C. Klingenberg. Zeilschrift Vereines Deulsch. Ing. 4 janvier, 11 janvier, 18 janvier, 25 janvier, w' février 1943.

La Victoria Falls and Transvaal Power $C^{0}$ se trouve dans la silualion, vraiment remarquable, de fournir 500 millions de Kwh par an au boul de seulement 4 années d'existence, et il est probable que cette production deva bientot s'blever ì t ooo millions dir hilowalls-heure.

Cette entreprise est done une des plus importantes du monde.

(1) A lexclusion des machines comportant des conducteurs rectangulaires massifs dans lesquels la perte par courants parasites dans le cuivre peut être importante.
Une partie importante de l'óncrgie est réparlie sur des distances assez considerables par le moven de l'air comprimí, ju ono che. vaux environ, étant utilisés de cette façon.

On a songé dès 1905 à utiliser la puissance des célèbres chules de Victoria. La Victoria Falls Power $\mathrm{C}^{0}$ fut constituée à ect effel et l'Allgemeine Elcklrikitäs (iesselschaft fut chargée de l'iquipe ment des stations génératrices.

Deux usines furent érigées dès le début, une à Brakpan, arẹ un équipement de $2 \times 3$ oou Kw., et une à Simmerpan arecu équipement de $4 \times 3000 \mathrm{kw}$. A Brakpan, les générateurs inrent livrés bobinés pour ro ono volts, mais ultérieurement on les rebolsina pour basse tension et on leur adjoignit des transfum. teurs portant la tension a so ooo volts. La raison de celte modjfication est que les bobinages haute tension placés direciement sur les alternateurs étaient détériorés par les decharoges atmenghi riques. - Le bobinage en hasse tension permit laugmenter la puissance des généraleurs de ro pour roo.

La distribution, dans le voisinage de Brakpan, est faite a la tension de 10000 volts. La tension est élevic à 40 ono volts pour un foeder allant dans la direction de Simmerpan.

L'usine de Simmerpan est équipé pour prodaire à une tensin de ro 000 volts l'énergie destinée an district et it une fension de 40000 volts celle alimenlant un feedor allant dans la direction de Brakpan. Entre ces deux stations est celle d' "Ilereule " où la tension est ramenéc a ro oon volts pour les besoins du district.

Indépendamment de cetle distribution emprunlée anx usines de Victoria Falls, il y a, aú Transvaal, d’importantes usines à vamur sur lesqueles larticle, abondamment illustre, donue des intications.

P. Boctrangivon.

\section{SOCIÉTÉ INTERNATIONALE DES ELEGITRIGIENS}

Proposition de la $2^{\text {e }}$ section sur les grandeurs et les unités photométriques, rapport de M. Bocuer.

Nous croyons intéressant de reproduire ici un rapport datant déjà de l'année dernière mais dont l'importance n'échappera pas a nos lecteurs.

La deuxième section du Comité de la Société avait chargé M. Bocier d'un rapport sur le résultat de l'examen des propositions relatives aux grandeurs et unités photométriques, qui ont été soumises à la Société internationale des Electriciens, et c'esl ce Rapport dont nous donnons la reproduction presque complete.

Cette question nous intéresse d'autant plus, que la photométrie est une science d'origine française dont les premières lois el les premières milhodes le mesures ont été exposées an xvm ${ }^{\mathrm{e}}$ side par l'illustre Bonguer et par Lambert.

La détermination des grandeurs et unités photométriques a déja écétudice par les physiciens à differentes reprises; elle a fait notamment l'objet d'unc importante discussion au Congris des Electriciens de Genève en $₫ 896$.

C'est au savant $\mathrm{M}$. Blondel que reviennent le mírite et lhonneur des améliorations considérables apportées à la terminologie photométrique, par l'application a la lumiere de définitions antlognes a celles usities pour le magnétisme. Ja consibíration dit llux lumineux qui domine ces réformes est particulièrement fertile en conséquences utiles.

Ces travaux ont conduit à l'elaboration, au Congres de Geneve de 1896 , d'un systeme cohérent ayant pour base la bougie décimale (qu'on supposail alors itre représentée avec une approximation pratique suffisante par l'étalon IIefner), le mète et la seconde. Depuis cette époque, différents changements sont survenus, parmi lesquels il convient de signaler on premiere ligne l'accord heureux qui s'est ctabli entre les Laboratoires nationatio des Bitats-Unis, de la France el de l'Anglelerre pour l'adoption d'une bougie internationale commune aux trois pars el dont la valeur est sensiblement la même que celle de la bougre décimale 
ou bougie Violle, laquelle est égale au r/20 de l'étalon au platine défini par M. Violle et a été adoptée comme unité photométrique par le Congrès international des Electriciens de Paris, en 1889 , sur sa proposition.

La comparaison de l'unilé Hefner a la bougie décimale a ćté, dautre part, l'objet de mesures très précises des différents laboratoires nationanx, et, en particulier, a été faite en France par le Laboratoire central d'Electricité (MM. Laporte et Jouaust, sous la direction de $\mathrm{M}$. Janet) et le Laboratoire national d'essai du Conservatoire des Arts-et-Métiers (M. Pérot). On a été conduit ansi à établir que l'unité Hefner est, pratiquement, les $0,9^{\circ}$ de la bougic intemationale, de sorte que loutes les mesures faites avec la hougie Hefner pcuvent être converties immédiatement en mesures internationales.

Le moment a donc paru venu aux spécialistes de différents pays de remettre au point le système international d'unités et de gran. deurs photométriques, el cette tâche a été entreprise aux ElatsLnis par l'llluminating Engincering Society, on rgro, sous la présidence de M. Humphreys. - Une sous-commission a été chargée de ces travaux. Rendant un juste hommage aux mérites de nos compatriotes, les Américains ont tonu à ce qu'ils fussent repré. sentés dans cetle sous-commission qui comprenait : MY. Sharp, président, Blondel, Kenclly, Nichols et Rosa.

Les définitions arrètés par ce Subcommiltee on Nomenclaturs and Standard et qui font l'objet de la proposition soumise a la Société internationale des Electriciens, sont portées au Tableau ri-annexé.

Ces définitions diffirent peu de celles qui ont été adoptées autrefois à Genève; on a préféré comme unité de longueur le centinètre au mètre, afin d'obtenir un plus grand caractère d'internationalité, élant donné que les pays anglo-saxons n'ont pas encore adoplé le système métrique, mais sculement le système C.G.S.

On a, d'autre part, admis comme unité la bougie internationale.

Enfin, on a rétabli la définition de la radiation spécifique que II. Blondel avait présentée autrefois à Genève et qui n'avait pas ité acceptée; l'expérience a démontré que cette radiation spécifique est fort utile.

Enfin, l'adoption du centimètre comme unité de longueur fondamentale a fait disparaître le lux ou hougie-mètre comme unité tondamentale d'éclairement au profit du lumen par centimètre carré ; unité qui a l'avantage de porter en elle-même sa définition.

Ce Tableau, qui constitue la mise au point probablement définitive des unités photométriques, figurera dans le Recueil des constanles de la Société de Physique. Toutefois, dans cette publication, le flux lumineux est représcnté par $\Phi$ et l'intensité lumineuse par J, suivant un usage déjà très répandı, afin d'éviter des onfusions avec la force et lintensité du courant.

La définition des grandeurs et unités photométriques est basce sur les considérations suivantes :

La lumiere se manifeste par l'excitation que produit la radiation qui provoque la vision. Le débit, ou flux de lumière, est proportionnel au flux de l'energic xayonnante et a un coefficient d'excitation qui dépend principalement de la distribution spectrale to cette énergie. La présence du flux de lumière se constate par l'elairement qu'il produit. L'éclairement est proportionnel au Sux par unité d'air de la surface ćclairée ou à la densité du flux tombant sur colle surface.

L'intensité lumineuse d'une source ponctuelle de lumière est mesuréc par lc flux lumincux émis dans un angle solide ćgal à l'unité suivant la direction dans laquelle l'intensité est mesurée.

Dewx sources ponctuclles de lumièrc so disent égales en intensilé quand elles produisent un éclairement égal à des distances égales.

Grâce au fait qu'on peut obtenir des sources ponctuelles étalonnées d'intensité lumineuse invariable, tandis qu'il n'est pas pratique d'obtenir un flux lumineux étalonné ou un éclairement étalonné sans avoir à sa disposition une source de lumière étalonnée, l'unité d'intensité lumineuse est devenue l'unité photométrique fondamentale dont dérivent les valeurs des unités de flux, l'ćclairement, elc. ; autrement dit, l'unité d'intensité est l'unité photométrique fondamentale, quoique le flux soit la grandeur photométrique fondamentale.
L'unité d'intensité lumineuse est la bougie. Par bougie, on culend la bougie commune à la Grande-Bretagne, à la France el a l'Amérique et à laquelle on a proposé de donner le mom de bougie internationale. Le terme paissance lumineuse peut the employé comme equivalent an terme intensiti lumineuse ch bougies.

Limité de flux lumineux est le fhux de lumière produit dans un angle solide égal à l'unité (un stéradiam) par une source mi. forme de lumiere de a bougie place au sommet de lingrle. Celle unile est appelée lumen.

le lumen est done indépendant des unités métrigues. Celle patrlicularité en a facilité l'adoption crénérale.

L'unité d'éclairement est l'éclairement qui est produit par un flux de a lumen tombant sur une surface plane égale a l'unite. Get colairement est éral à celui qui est produit par ume somec do I bougie placée à l'unité de distance. L'unité d'éclairement dans le systène C. G.S. est le lumen par centimètre carré. Comme unité pratique, on recommande le millilumen par centimelre carré qui est égal à 10 bougies-mitres, on à I lumen par metre carré, ou bien à of flux, terme qu'il serait intéressant de conserver.

Par intensilé spécifique d'une surface ou corps hmineux, ex. pression qui remplacera disomais avantageusement celle heaucoup moins claire d'éclat intrinscique, on entend, suivint les principes générax de la nomenclature physique, me inlensibé lumineuse apparente par unite de surface expriméce en bougies par centimètre caré de surfare apparente, c'est-it-dire de surface projetéc sur un plan perpendiculaire a la direction suivant latquelle elle est vike par lolservateur. Cela en considerant sculement une surface de dimensions petites par rapport à la distamee ì laquelle esl faite la mesure. 11 ch résulte que pour les surfaces lumineuses dont l'emission suit la loi du cosinus, lintensilí spécifique est indépendante de la direction de visée.

Par radiation spécifique d'une surface ou corps luminems, on entend son flux lumincux folal par unite de surface exprimes on lumens par centimidre arre ou en millilumens par cenlimidre carré. Pour les sutfaces lumineuses dont l'émission suit la loi du cosinus, la radiation spexifique est égale a l'inteusité sprécifique multipliée par $\pi$.

Par intensité moyenne sphérique d'une source do lumière, on entend la valeur moyemne de son intensite mesurée dans toules les directions de l'espace ; clle est égale au quotient du flux lumineux total exprimé en lumens par $4 \pi$.

Par facteur de réduction sphérique d'une source de lumière, on entend le rapport de son intensité moyenne sphérique ì son intensité moyenne horizontale.

les noms de ces unités dans lo système G, G.S. et leurs relations malhématiques entre clles sont risumécs dans ce Tablenn :
Grandeurs pihotométriques
Nom de l'linite
Eluation de cléfiutlon
1. Intensité de lumiè- ?
re oul pilissance de re ou pilissance de
source lumineuse.
2. Flux lumineux....., l.umen.
4. Eclairement........ Radiation spicillque. $\frac{\text { Lumens Millilumens }}{\mathrm{cm}^{2}}$
5. Intensité spécilquue. $\frac{\text { Bougies. }}{\text { crín }^{2}}$.
6. Intensite spécifique. Immen-heurooulumen $Q=F E$. scoonde.

La bougie internationale est representée cn abréviation par $C$; Le lumen internalional est représentí en abréviation par $\mathrm{J}$;

l'unité Hefner est reprisentée en abréviation par HK, suivant l'usage actuel de l'Allemagne;

$f$ est le facteur de réduction sphérique dune source de lumior: ; w est un angle solide égal à aire diune sphère sous-tendant un angle;

$r$ est la aistance de la source à la lumière en centimetras; $m$ est lecoefficient de rélexion ou de radiation $(1-m=$ absorption : $S$ est une aire mesurée en centimetres ;

$c$ est un angle d'emission;

$i$ est un angle dinciulence:

(1) $S$ se rapporte a la surface vue du point auquel l'intengité 1 est rapportée. 
Les membres de la deuxième section ont unanimement approuvé le Tableau des grandeurs photométriques proposé sous les auspices de l'llluminating Engineering Society. Ils ont été heureux d'y louver une nouvelle consécration des idées des physiciens frangais par une association magistralement organisée, au sein de laquelle ces étuds photométriques ont été longuement poursuivies dans un esprit scientifique des plus élevés.

\section{NOTES ET INFORMATIONS}

\section{International Engineering Congress, 1915.}

A l'occasion de l'Exposition Internationale du Panama-Pacifique, un Congrès international d’ingénieurs aura lieu à San-Francisco, en 1915, auquel les ingénieur's du monde entier seront invités à participer.

Le Congrès est placé sous le palronage des cinq Sociétés nationales d'ingénieurs suivantes : "American Society of Civil Engineers ", "American Institute of Mining Engineers", "The American Socicty of Mechanical Lngineers ", "American Instilute of Electrical Engineers " et "The Society of Naval Architects and Marine Engineers $n$.

Ces Sociétés, dans un esprit de coopération, ont nommé un comité permanent d'organisation, se composant des présidents et des secrétaires de chacune de ces sociétés, et de dix-huit membres domiciliés à San-Francisco.

Ainsi constitué officielleutent, le personnel du Comité se com. pose des membres suivants :

American Sochety of Givif. Exgneers. - Geo. F. Swain, Président ; Chas. Warren Hunt, Sccrétaire ; Arthur L. Adams ; IV.-A. Callell ; Chas. Derleth, Jr. ; Chas. D. Marx.

Anerician Instrutue of Mining Begreers. - Charles F. Rand, Président : Bradley Sloughton, Secrétaire; H.-F. Bain ; Edw. H. Benjamin ; Newton Cleaveland ; Wm. S. Noyes.

Tie Americay Sogrety of Mecuavical Engrneers. - W.-F.-M. Goss, Président; Calvin W. Rice, Secrétaire; W.F. Durand; R.-S. Moore ; T.-W. Ransom ; C.-R. Weymouth.

American Institute of Electrical Engineers. - Ralph Daven. port Mershon, Président ; F.-L. Hutchinson, Secrétaire ; J.-G. De Remer ; A.-M. Hunt.

Tile Society of Naval Architects and Marine Engrneers. Robert M. Thompson, Président ; D.-H. Cox, Secrétaire ; Geo. W. Dickie ; W.-G. Dodd ; Wm.-R. Eckart ; H.-P. Frear.

Ce Comilé s'est organisé d'unc façon définitive, a élu pour président : Prof. W.-F. Durand, ef pour secrétaire-trésorier : W.-A. Caltell, ct a ouvert les bureaux du Congrès à l'adresse suivante : Foxcroft Building, 68 Post St., San-Francisco.

Les dix membres du Comité, qui sont les présidents et les secrétaires des cinq Sociétés nationales, constituent le Comité de Participation, qui aura charge de lancer toutes les invitations à prendrc part au Congrès, aux gouvernements, aux sociétés d'ingénieurs et aux membres.

Ce Comité se décompose comme suit :

Comrté de participatios. - Chas. F. Rand, Président ; Chas. Warren Hunt, Secrétaire; D.-H. Cox ; W.-F.-M. Goss ; F.-L. Hutchinson ; Ralph Davenport Mershon ; Calvin W. Rice ; Bradley Stoughton ; Geo F. Swain : Robt. M. Thompson.

L'organisation du Congrès ainsi que la tâche d'obtenir et de publier les rapports seront laissés aux soins des membres du Comilé domiciliés à San-Francisco. Le travail a été réparti entre divers sous-comités, et le président Durand a désigné les membres pour ces sous-comités comme suit :

Comité exécutrF. - W.-F. Durand, Président, ex-officio ; W.-A. Cattell, Secrét.; E.-H. Benjamin ; W.-G. Dodd ; A.-M. Hunt.

Conuté des Finances. - W.-G. Dodd, Président ; Newton Cleaveland; R.-S. Moore.

Comité des Rapponts. - A.-M. IIunt, Président ; A.-L. Adams ; H.-F. Bain ; G.-W. Dickie ; W:-R. Eckart ; C.-D. Marx ; C.-R. Weymouth.
Comirí de Publicrté. - W.-A. Cattell, Président ; C. Derleth, Jr. ; W.-S. Noyes ; T.-W. Ransom.

Conté local. - E.-H. Benjamin, Président ; J.-G. De Remer; H.-P. Frear.

Le bureau honoraire du Congrès se composera d'un président et de vice-présidents choisis parmi les ingénieurs les plus émi. nents des divers pays représcntés au Congrès.

Les rapports présentés seront répartis entre divers groupes ou sections. Pendant le Congrès, chaque section se réunira séparément sous la présidence d'un membre, reconnu pour sa compé. tence dans les branches spéciales représentées par cette section.

Les détails du Congrès noont pas encore été déterminés, mais le but vrai est d'y présenter les méthodes pratiques les plus $\mathrm{m}$. ployées dans le monde enticr, et les rapports, les discussions ef lcs comptes rendus composeront une revue détaillée des progrìs accomplis dans les demieres dix années et un ensemble des déve. loppements les plus récents et des méthodes les plus justifícs lans les diverses branches de la science d'ingénieur.

Les rapports groupés et publiés par le Congrès formeront une collection d'une valeur inestimable, et la publication s'en fera a in tel prix et de telle manière que le plus grand nombre puisse se les procurer.

Les différents Comités sont à l'cuvre et, sous peu, des rensejgnements supplémentaires seront publiés quant aux cotisations, programmes, etc...

\section{L'adduction des eaux à Los Angeles (1).}

Los Angeles, l'un des plus jeunes villes du Nonveau Monde, en est aussi l'ume des plus entreprenantes. Métropole de la Californie méridionale, elle avait vu sa population augmenter si rapidement, que ses ressources en eau potable étaient devenues insuffisantes moins de 26 ans après sa fondation.

Ses ingénieurs avaient vainement cherché des sources dans les environs, et l'on désespérait déjà de trouver une solution à ce problème d'un intérêt vital, quand un homme audacieux proposa de capter les eaux limpides d'une rivière distante de 4 ro kilo. mètres et séparée de la ville par la chaîne de la Sierra-Nevada et par le désert de Mohave.

Le projet fut mis à l'étude en rgo', et, tout d'abord, la municipalité recula deyant les responsabilités qu'on lui demandait d'as. sumer : l'entreprise devait entraîner une dépense de près de 25 millions de dollars, soit i 25 millions de francs. Comme la ville comptait r52 000 âmes, c'était 800 francs de dettes qu'on demandait à chaque habitant de contracter en échange d'une distribution illimitée d'eau pure.

Mais, pour Los Angeles - la Ville des Anges - cela devenail une question de vie ou de mort. L'emprunt fut contracté, et les ingénieurs se mirent aussitòt à l'cuvre.

Le premier coup de pioche fut donné vers la fin de rgo6. Avant tout, on se préoccupa d'alimenter en eau potable l'armée de 4 ooo ouvriers répartis en différents camps dans le désert de Mohave, ce qui motiva la construction de quatre réservoirs of la pose de 305 kilomètres de conduiles. Ces ouvriers établirent un réseau téléphonique de 650 kilomètres, préparèrent 485 kilomètres de route pour assurer le transport du matériel, et construisirent quatre usines hydroélectriques. Simultanément on éleva une usine à fabriquer le ciment, au coût de 5 millions de francs.

Ce n'étaient là que des travaux de préparation ; ils durèrent dix-huit mois. Enfin, en octobre 1908 , le véritable travail de construction commença, et se poursuivit dirant plus de 4 ans a raison de 80 kilomètres de longueur par année. Celte curre gigantesque, qui fait le pluṣ grand honneur aux ingénieurs callforniens, approche de son achèvement, et il est probable qu'elle sera inaugurée d'ici quelques semaines.

L'eau est captée à une altitude de $\mathrm{x}$ 27 $\mathrm{r} \mathrm{m}$. environ au-dessus du niveau de la mer. Elle s'écoule d'abord par un canal à ciel ouvert long de $36 \mathrm{kms}$, puis par un canal de $60 \mathrm{kms}$ construt en ciment. Elle franchit ensuite, par la force de gravité, une dis.

(1) Nous reproduisons cette information parue sous la signaturede M. V. Fortis, dans l'excellente revue : La Nature. 
est de $\mathrm{o}^{\mathrm{m}} 83$. Elles sont assez bien défendues pour pouvoir faire la traversée maritime de Port-Saint-Louis à Marseille.

Chaque remorqueur devra pouvoir remonter quatre bar¡ues d'Arles à Lyon dans un délai ne devant pas dépasser quatre-vingts heures de marche, chaque barque étant chargée à un tirant d'eau maximum de $\mathrm{x}^{\mathrm{m}} 80$; en cas d'insuf-. fisance d'eau sur les seuils, le tirant d'eau des barques devra êtrc inférieur de $\mathrm{o}^{\mathrm{m}} 2 \mathrm{O}$ au mouillage assuré.

Tout ce matériel est actuellement en construction et commencera ses essais à l'automne prochain. Il n'est pas douteux (que, s'il donne les résultats qu'on en attend, le problème économique de la navigation sur le Rhône aura fait un grand pas en avant.

\section{Trafic du Rhône}

Il n'existe aucune statistique officielle du trafic du fleuve antérieure à 1847 ; depuis cette époque jusqu'en I88I, date de la suppression des droits de navigation, on ne trouve que celle, assez incomplètc d'ailleurs, de l'Administration des Contributions indirectes, chargée de percevoir les droits.

A partir de r881, la statistique du Ministère des. Travaux publics fournit les renseignements les plus complets et les plus précis.

Quoi qu'il en soit, M. Lavaud donne quelques chiffres anérieurs à 1847, extraits des études publiées par MM. Armand et Voisin, ingénieurs en chef.

Ces chiffres montrent notamment que de Lyon à Arles 1287 kilomètres) le tonnage total - descente et remonte a passé de 420 ooo tonnes en I 830 à 236295 tonnes en I 9 ro. Le maximum : $4965 \mathrm{rg}$ tonnes, a été atteint en $\mathrm{r} 855$, et le minimum : 122412 tonnes, s'est produit en r88o. La décroissance a été surtout sensible de 1860 à $\mathbf{1} 885$ et le relèvement entre igoo et $\mathbf{1} 905$.

\section{LAMÉLIORATION DE LA NAVIGATION}

\section{ENTRE LYON ET ARLES}

\section{Concours de 1 Office des Transports}

But ex organisatron pu Concours. - Tels étaient l'état du lleuve et la situation de la navigation lorsque le Comité Directeur de l'Office des Trassports, qui groupe vingt-huit Chambres de commerce de la région du Sud-Est, adopta, dans sa séance du 23 juin rgo8, tenue à Grenoble, le principe d'un concours destiné à encourager les études de toutcs sortes tendant à une meilleure utilisation du fleuve.

Dès cet instant, M. R. Tavernier, ingénieur en chef des Ponts et Chaussées, l'émincnt ingénieur-conseil de l'Office, lit remarquer que si l'utilisation dite "intégrale n, c'est-à-dire au triple point de vue de la navigation, de l'irrigation et de la production de la force motrice, était, seule, de nature à donner satisfaction à tous les desiderata, les dépenses à envisager étaient tellement considérables" que ce n'était pas relarder l'exécution de l'ouvre, mais plutôt la faciliter, que d'admettre la possibilité de la poursuivre par étapes.

Les diver's tronçons du Rhône, entre Lyon et Arles, ne se prêtent pas également bien à l'aménagement des forces hydrauliques et sur certains points des canaux mixtes, semblables à ceux de Jonage, pourront être réalisés.

Le principe du concours était ainsi établi :

Il était impossible de le limiter aux seuls projets d'aménagement intégral de tout le parcours, car on risquait ainsi d'écarter un certain nombre d'idées ingénieuses; on devait, au contraire, y admettre des projets spéciaux, s'appliquant à certains tronçons et susceptibles de démontrer la valeur d'une solution plus particulièrement applicable à ce tronçon.
Il ne pouvait s'agir d'arrêter les détails d'exécution d'un projet mais plutôt d'orienter, vers les solutions réalisables, un courant d'opinion, puissant et unanime quant au but $\dot{a}$ atteindre, mais, jusqu'ici, très divisé quant aux moyens à employer.

Il s'agissait, en somme, non d'un concours de projets définitifs, mais bien d'un concours d'idées.

Dans sa séance du 28 juin r 909 , le Comité directeur de l'Office des Transports décida de faire appel non seulement aux Chambres de commerce, mais encore aux municipalités, collectivités et même sociétés intéressées, de manière à constituer un fond de Concours assez important pour susciter des participations sérieuses.

Puis une Commission d'organisation de concours fut nommée qui désigna $M$. Coignet pour son président.

Dans sa séance du 9 juin rg Io, cette Commission eut la satisfaction de constater que la louable initiative de l'Office des Transports avait porté ses fruits; une somme 14: 52 650 francs était souscrite.

Elle arrêta le programme du Concours tel qu'il a été reproduit ici ( $\mathrm{N}^{\circ}$ de Mars $\mathrm{I}_{9} \mathrm{r}_{2}$ ), en tête du rapport de M. Côte.

(Suivent, dans le travail de M. LAvaud, la composition du Jury et la liste des concurrents ; ayant déjà publié ces documents, nous ne les reproduisons pas.)

D’une façon générale on pouvait classer ainsi les avantprojets soumis au Jury :

$\mathrm{r}^{\circ}$ Projets n'envisageant que la navigation, soit par canal latéral, soit en empruntant partiellement le lit du fleuve ;

$2^{\circ}$ Projets de navigation avec utilisation de la force motrice produite par des usines-barrages ou des usines établies sur dérivation ;

$3^{\circ}$ Projets de navigation dans le lit du fleuve avec création, dans les vallées secondaires, de réservoirs d'arrêt permettant à la fois de régulariser le débit du fleuve et de produire de la force motrice.

Le cadre de ce rapport ne permet pas de fairc un résumé de chacun des projets présentés ; seuls les projets primés pourront être analysés; ce sont les suivants :

$$
\begin{aligned}
& x^{\text {er }} \text { Prix, } 20 \text { ooo fr. : MM. BrLlet et Givoiset, à Lyon. } \\
& 2^{\circ} \text { Prix } \mathbf{1} 2000 \mathrm{fr} \text { : : M. Mollard, à Paris. } \\
& \text { ex-equo } \text { I } 2 \text { ooo fr. : Soc. des Grands Travaux de Marsetlue } \\
& 3^{\circ} \text { Prix, } 4000 \mathrm{fr} \text {.: M. Mournallee, à Lyon. }
\end{aligned}
$$

\section{Projet de MM. Billet et Givoiset}

Les auteurs font observer que, sur la plupart des seuils situés dans la section comprise entre les confluents de l'Ardèche et de l'Isère, la vitesse du courant, à l'étiage, atteint 4 à 5 mètres à la seconde, tandis que, sur les rapides des autres sections du fleuve, la vitesse ne dépassc guère, el en quelques points seulement, 3 mètres, soit I $\mathrm{I}$ kilomètres à l'heure. Or, cette vitesse qui, d'ailleurs, pourrait, sans doule, être encore diminuée par quelques travaux, n'a ricn d'excessif ; c'est celle que l'on rencontre ì Bingen, sur le Rhin, où le trafic est très intense.

On voit donc que l'état actuel de navigabilité du Rhône. est excessivement favorable à la navjgation de descente, et que celle de remonte peut s'effecluer facilement, sauf sur la section comprise entre l'Isère et l'Ardèche.

Projet d'amélioration proposé. - Le projet d'amélioration du Rhône, à l'aval de Lyon, consiste simplement dans la création de dérivations éclusées, de faible longueur et à l'aị' libre, permeltant à la navigation de remonte d'éviter ces rapides, en général très courts. On rend ainsi la section dif- 
Barbizlon. - Laboratoire d'essais pour turbines hydrauliques, année rgo7, numéro de novembre, pages $24 \mathrm{x}$ et suivantes.

Comte de Sparre. - Des effets de résonnance qui peuvent se produire pour les hautes chates dans les coups de bélier, année 1907 , numéro de septembre, pages 203 dे 207, et numéro de décembre mème année, pages 277 à 279 .

A. Blancher. - Le renforceur de chutes, année igo8, numéro de novembre, pages $2 / 3$ et suivantes.

La Rédaction. - Amortisseur de coups de bélier à l'usine hydroélectrique du Tumwater Canyon, année 1909, numéro de novembre, pages 209 à 30 r.

A. Revard. - Note sur les modes d'action de la correction de réglage dans la régulation des lurbines, année 1909 , numéro d'août, pages 200 à 204 , et numéro de férrier de la même année, pages 40 à 46 .

A. Boucher. - Jaugeages par titration, année rgro, numéro de novembre, pages 284 et suivantes.

G. Senvirre. - La station d'études de turbines de Rioupéroux, année igro, numéro de janvier, pages 5 et suivantes.

Comte de Sparre. - Note au sujel de l'emploi des réservoirs d'air pour atténuer les coups de bélier dans les conduites forcées, année rgir, numéro d'octobre, pages ro7 et suivantes; numéro de novembre, pages 293 et suivantes; numéro de décembre, pages $3 \mathrm{r} 6$ et suivantes.

\section{HIBI IOGIRAPATE}

Les propriétés optiques des Solutions, par C. Cúnevenu, librairie Gauthier-Villars.

Certains chimistes ont, ì lhenre actuclle, tondance a mouler les conséquences de leurs observations dans une matrice mathé matique. Ils y sont tout naturellement incités par la parenté intime cui existe entre certains faits, dont les caractères chimiques cl physiques yont en quelque sorte se confondant, sans que la démarcation entre les faits ò la nature du corps s'altère et ceux où clles ne s'altère pas soit bien nette. Les mémoires qu'ils écrivont, sous l'empire de celte discipline intellectuelle, ont tout l'as. pecl de mémoires de phỵsique mathématique.

Le livre que M. Chéneveau, docteur ès-sciences, chef de travaux pratiques à la Faculté des Sciences de l'Université de Paris, próscnte aujourd'hui cst dans ce cas. C'est, l'auteur a soin de nous (n avertir dans son introduction, seulement une partie (importante à la vérité) d'un traité à faire sur l'optique des solutions, qui devrait comprendre en plus linterprétation des phénomènes d'absorption et de polarisation.

Nous espérons que, quelque jour, ce jeune savant donnera cetle suite nécessaire à cette thèse de doclorat écrite dans un esprit synthétique fort intéressant et qui, pour l'instant, se borne a l'étude des phónomènes de rúfringence produits dans des com. posis variés, ce qui ne veut pas dire que le champ ainsi exploró no soit pas trì's vaste. Partant des lois générales posées dans ces dernicrs temps par divers savants pour lier les indices de réfraclion aux propriétés moléculaires des corps, lois dont il donne des juslifications ingénieuses, il arrive à différentior les solutions bs phus diverses, et mòme à donner le moyen de se rendre compte s'il y a ou non combinaison au sein d'une solution.

T. travail se termine par une description tris methodique des appareils propres aux mesures de la refraction et de la dispersion dans les cas les plus divers. Une table de résultats numériques suil cette description et, dans une dernière partie, est indiquée la bihliographie, déjà très copicuse, des mémoires sur ces sujets.

Bien que l'application industrielle des jdés de $\mathrm{N}$. Chéneveau n'apparaissc pas avec une flagrante évidence, je crois cependant devoir signaler son travail aux ingénieurs qui, dans leur laboratoire, peuvent avoir des recherches à faire sur les solutions mises en envre dans leur industric. S'ils ne trouvent pas tout de suite dans le travail de M. Chéneveau réponse aux questions qui les préoccupent, peut-être trouveront-ils la quelque utile suggestion les mettant a mêmẹ de dégager l'inconnue cherchéc et peut-être des découvertes nouvelles.

Commandant Avnebrand.
Annuaire du bureau des longitudes pour Ian 1913, in-16 (16-9) de 883 pages, avec 11 figures et 1 planche. Prix net : $1 \mathrm{fr} .30$, cartonné 2 fr. Gauthier-Viliars, éditeurs, quai des Grands. Augustins, 55, Paris (6e).

L'Avertissement de l'Fditeur expose très clairement les nolices qui ont été spécialement développées cette année.

L'Annuaire " propre à régler ceux de toute la République ", dont la publication rentre dans les attributions du Bureau des Longitudes, parut, pour la premiere fois, en $179^{6}$; il se rappor. tait à l'an V, $179^{6-1797}$. Le présent volume est donc le $115^{\circ}$ de la collection.

Depuis rgoo, toutes les dates et heures sont exprimées en lemps civil moyen, compté de o h. à 24 h., à partir de minuit.

Dans le présent volume, par application de la loi du 9 mars I 9 II, les heures des divers phénomènes sont exprimées en temps légal, c'est-à-dire au temps moyen de Paris diminué de 9 minuies $2 \mathrm{I}$ secondes.

Conformément aux nouvelles dispositions adoplées en 1904 , le présent Annuaire contient des Tableaux détaillés relatifs à la Mé. trologic, aux Monnaies, à la Géographie et à la Statistigue, ảnsi qu’à la Météorologie et ne contient pas de données physiques el chimiques. Ce sera le contraire pour l'Annuaire de igrí.

En vertu de ce mème principe, la partie astronomique du pré. sent Annuaire renferme les tableaux relatifs à la déviation de la verticale en France, à l'intensité de la pesanteur en divers lieur et au calenl des allitudes par les observations barométriques : i] contient aussi les parallaxes stellaires, les étoiles doubles, les monvements propres et la spectroscopie stellaire, tandis qu'on y a sup. primé la sismologie, les cadrans solaires, la physique solaire et le' hbleau des petiles planètes : cos matières seront développles à nourean en rgí.

Voici maintenant les principales modifications apporties cette année à la rédaction de l'Annuaire.

Partic astronomique. - Comme déjà en Igr2, les matièrcs qui composent celle parlie ont été présentées dans un ordre plus r. tionnel, et. la rédaction des notions fondamentales de l'astronomic a été entièrement refondue par M. Andoyer, qui a revisé aussi les tableaux numériques correspondants; les notions indispensables sur les calcndriers en usage ehez les divers peuples civilisés sont dues in M. Rocques Desvallées ; les tables pour le calcul des hat teurs par les observations barométriques sont celles publises en 1806 par N. Angot dans les Annales du Burean Central méléoro logique, et adoptées par la Commission internationale d'aérosta tion scientifiques; les tables relatives a la réfraction ont été tirécs des tables plus etendues de R. Radau; NI. P. Puisenx a bien ronlu se charger de l'article sur la constitution physique de la Lune ; ha notice très détaillée sur les comètes apparues en ron a été rédigét commo d'habitude par MI. Schulhof, qui a revu aussi les éléments des satellites : le tablean des parallaxes stellaires a été revisé ef étendu par M. Bigonrdan, de façon à présenter l'ensemble des résultats les plus récents jour toutes les étoiles dont la parallare parait supéricure à un dixième de seconde; enfin M. de Gramont a bien voulu revoir son importante nolice sur la spectroscopic slellaire.

Géogrophie ef Statislique. - Les tahloanx qui sc rapportent à la géographir statistiquo ont ité mis à jour sons la dirertion de M. Rocques Desvallées : sans insister sur quelques nouvelles alditions de détail, signalons sculement que pour la France el l'tl geric on a introduit les résullats complets du reconsement it I9r derniers dénombrements connus.

Métrologie. - Dans cette partie, on a revisé la table des me sures anglaises comparécs aux mesures métriques d'après l'd de I 897 ; in tableau correspondant pour les mesures des Elats-Uni a élé tiré des Smilhsonian Physical Tables (rgro). Enfin M. li lietr tenant de vajsseau Tillier a bien voulu nous communiquer ume note détaillée sur le tonnage des navires.

L'Imprimeur-Gérant : P. Legendab 